\title{
Algunas consideraciones sobre la evolución de la formación ambiental de los maestros en formación del nivel básico.
}

\section{Some considerations on the evolution of the environmental formation of teachers in formation of the basic level}

\author{
1. Lubis Carmita Zambrano Montes \\ 2. Marylú Núñez Morales
}

\section{Resumen}

El presente trabajo persigue como finalidad valorar la evolución histórica de la formación ambiental que han recibido los maestros en formación del nivel básico de educación, con especial énfasis en el Ecuador, a partir del análisis de las concepciones que han existido sobre esta dimensión de la educación a través de su historia, y los grandes eventos y foros internacionales que han sentado pautas en la materia. En ese orden, se tienen en cuenta tres etapas de desarrollo de la Educación Ambiental, la primera de ellas denominada Concepción naturalista de la educación. (Hasta la década de los años 70 siglo XX), la segunda Desarrollo de la concepción de la educación ambiental, (entre los años 1970 al 1992) y la tercera de Redimensionamiento de la educación ambiental (del año 1992 hasta la actualidad).

\section{Palabras clave}

Etapas de la educación ambiental, formación de profesores, desarrollo sostenible, competencias.

1. Instituto Superior Pedagógico "23 de Octubre". Montecristi - Manabí- Ecuador. Calle Manta y el Porvenir 2310053. Email - instituto23deoctubre@hotmail.com

2. Universidad de Camaguey Ignacio Agramonte Loynaz - carretera Circunvalación Norte Km.5.5 Camaguey Cuba teléfono (53) (32) (26 18 69) marylu.nunez@reduc.ed.cu 


\section{Abstract}

The present work pursues like purpose appraising the historic evolution of the environmental education that the teachers in formation of the basic level of education, with special emphasis in the Ecuador, as from the analysis of the conceptions that have existed on this dimension of education through its history have received, and grand events and international forums that have established guidelines in the matter. In that order, they have in account three stages of development of environmental education, the first theirs named naturalistic Conception of education. (To the decade of years 70 century XX), second Evolution of educational environmental conception, (enter years 1970 to the 1992) and the third class of new conception of environmental education (of the year 1992 to the present time)

\section{Keywords}

Stages of environmental education, teacher training, sustainable development competencies. 


\section{Introducción}

En la misma medida que crece el impacto de la civilización humana el medio ambiente y la problemática ambiental fue haciéndose cada vez más perceptible, empiezan a aparecer también signos de la formación de una conciencia sobre la necesidad de proteger la naturaleza; con ello comienza la preocupación por desarrollar acciones educativas con ese propósito. Así nace el término educación ambiental. Novo (1998). Surge entonces como un imperativo la formación inicial y permanente de profesores de todos los niveles de educación para poder asumir por las vías formales la Educación Ambiental de los niños y jóvenes.

La concientización para la educación ambiental debe ser asumida primordialmente por la educación, por lo que es necesario que los docentes se formen para orientar a los estudiantes hacia la reflexión y prácticas que apunten al desarrollo sostenible del medio en función de satisfacer sus necesidades, sin menoscabar las de las futuras generaciones.

Por lo que se persigue como finalidad en este trabajo valorar la evolución histórica de la Educación Ambiental que han recibido los maestros en formación del nivel básico de educación, con especial énfasis en el Ecuador, a partir del análisis de las concepciones que han existido sobre esta dimensión de la educación a través de su historia, y los grandes eventos y foros internacionales que han sentado pautas en la materia. 


\section{Desarrollo}

La evolución de la formación ambiental de los maestros del nivel básico de educación no debe valorarse al margen de la historia de la propia Educación Ambiental, donde en esta última se pueden delimitar tres etapas marcadas por los cambios esenciales de las principales concepciones que han existido sobre la misma, la primera de ellas denominada Concepción naturalista de la educación. (Hasta la década de los años 70 siglo XX), la segunda Desarrollo de la concepción de la educación ambiental, (entre los años 1970 al 1992) y la tercera de Redimensionamiento de la educación ambiental (del año 1992 hasta la actualidad) (Núñez Morales M. 2003)

Primera etapa: Concepción naturalista de la educación. (Hasta la década de los años 70 siglo $X X)$.

Con independencia de que el reconocimiento en términos oficiales de la educación ambiental, no es hasta la década de los años 70 del pasado siglo, es preciso partir de sus antecedentes, pues desde la antigüedad existe tradición del uso del medio como instrumento didáctico y un deseo explícito de educar en la naturaleza (Núñez Morales M. 2003, 2012). Ésta se considera como una fuente de conocimientos y de formación para los niños. Así, para Rousseau (17|2-1778), la naturaleza es nuestro primer maestro; para Freinet (1963) la enseñanza de las ciencias tendría que basarse exclusivamente en la observación y la experiencia infantiles en el mismo medio. 
En esta etapa la educación relacionada con el medio constituye un grupo de acciones aisladas con el fin de proteger el entorno natural, sin existir programas institucionalizados con tal objetivo, y asociada sobre todo a la enseñanza de las ciencias naturales.

En ese orden, en el Ecuador en las primeras décadas del siglo XIX, con la influencia de la Revolución Industrial, del Positivismo y del Pragmatismo, se producen innovaciones en el sistema educativo: El proceso de formación del hombre trata de ser incorporado al desarrollo social, haciendo abstracción de la visión idealista y estática del mundo y la sociedad. Esta concepción ideológica planteó determinados pre-requisitos, siendo alguno de ellos: la libertad educativa, la cual sostuvo que el único conocimiento válido es aquel que tiene una función utilitaria, y el diseño en la programación educativa del tratamiento de las ciencias, la experimentación, el conocimiento práctico y la investigación de la naturaleza.

El nacimiento del movimiento denominado educación ambiental comienza a finales de la década de los años 60 , se toma como punto de referencia la fundación del Council for Environmental Education -Consejo de Educación Ambiental- en la Universidad de Reading, Inglaterra (año 1968), organismo de carácter planificador y coordinador, que pretendía aglutinar e impulsar el naciente trabajo que, sobre el medio ambiente, estaban desarrollando algunas escuelas y centros educativos del Reino Unido.

La educación ambiental en esta época se caracteriza por tener un claro tinte conservacionista; "...las experiencias pioneras se relacionan con itinerarios y 
actividades en la naturaleza, salidas al campo etc., impulsadas por grupos de maestros y profesores innovadores e inquietos..." (González Muñoz M. 1998: 2).

Las primeras definiciones lograron la construcción del término como un proceso educativo que se ocupa de la relación del hombre con su entorno natural y artificial, incluyendo la relación de la población, la contaminación, la distribución y el agotamiento de los recursos, la conservación, el transporte, la tecnología y la planificación rural y urbana con el medio humano total (Valdés Valdés O. 2002).

Sin embargo, producto de las propias limitaciones de la concepción del medio ambiente en esos momentos, en la definición de educación ambiental antes vista, no se tienen en cuenta aspectos del medio tan importantes como los sociales, los éticos y los estéticos, los que a la luz de posteriores análisis, pasan a ser elementos vitales de la educación ambiental. (Núñez Morales M. 2003,2012)

Segunda etapa: Desarrollo de la concepción de la educación ambiental. (1970 1992).

A partir de las décadas de los años 70 y 80 del pasado siglo, producto del agravamiento de los problemas ambientales, se efectúan a nivel internacional toda una serie de foros y convenciones, los que sientan pautas para el desarrollo de la educación ambiental.

Especiales aportes se hacen a la educación ambiental en la Conferencia de Naciones Unidas Sobre Medio Ambiente Humano, celebrada en Estocolmo, Suecia(1972), donde se insta al desarrollo de la educación ambiental como uno de 
los elementos más importantes para enfrentar la crisis ambiental mundial. En este período (1972) es creado el Programa de Naciones Unidas para el Medio Ambiente (PNUMA) el que ha jugado un importante papel en el desarrollo de la educación ambiental, al estar dentro de sus objetivos la educación y capacitación en esta materia.

En la 1era Conferencia Intergubernamental Sobre Educación Ambiental, celebrada en Tbilisi (1977), la que establece las orientaciones para su incorporación al sistema educativo, desde la educación no formal y los niveles de instrucción básica, hasta la formación universitaria, y que en cuanto a la formación docente plantea "La formación de personal cualificado se considera una actividad prioritaria. (....). Es evidente que ni los mejores programas de estudios ni el mejor material pedagógico podrán tener el efecto deseado si quienes están a cargo de los mismos no han asimilado los objetivos de la Educación Ambiental y si no son capaces de llevar a cabo el aprendizaje y las experiencias que la misma comporta, ni de utilizar eficazmente el material que tienen a su disposición" (1978: 7); y en su informe final se define la educación ambiental como:

"La reorientación y articulación de las diversas disciplinas y experiencias educativas que facilitan la percepción integrada del medio ambiente, haciendo posible una acción más racional y capaz de responder a las necesidades sociales. Tiene por objetivo transmitir conocimientos, formar valores, desarrollar competencias y comportamientos que puedan favorecer la compresión y la solución de los problemas ambientales" 
Por su parte, en el Primer Congreso de Educación Ambiental celebrado en Moscú (1987), se tratan aspectos tales como la información, investigación, experimentación de contenidos y métodos, y la formación del personal docente, entre otros. "La formación del profesorado es un factor clave para el desarrollo de la Educación Ambiental " (1987: 20); en el informe final se definió la educación ambiental de la forma siguiente:

"La Educación Ambiental se concibe como un proceso permanente en que los individuos y la colectividad cobran conciencia de su medio y adquieren los conocimientos, los valores, las competencias, la experiencia, capaces de hacerlos actuar individual y colectivamente para resolver problemas actuales y futuros del medio ambiente".

La educación ambiental pasa de educación para proteger a la naturaleza, a educación para proteger el medio ambiente, considerando al mismo en su totalidad, es decir, en sus aspectos naturales y en los creados por el hombre, así como los tecnológicos, sociales, éticos y estéticos "Una «conquista» que ahora puede parecernos lejana pero que requirió de largos debates y procesos, consistió en ampliar el concepto de medio ambiente, que hasta ese momento estaba asociado casi exclusivamente al medio natural" (Novo Op. Cit. P.6) En el tratamiento conceptual dado a la educación ambiental en este período, se pone de manifiesto la ampliación de su concepción. 
A partir del análisis efectuado de la educación ambiental desde su surgimiento hasta la década de los años 80 del pasado siglo, se puede concluir que:

- Desde su origen se le ha conferido una gran importancia a la preparación de los maestros y profesores para que asuman la educación ambiental de sus estudiantes.

- La concepción de la educación ambiental evoluciona en esta propia etapa pues:

A) Se llega a determinar su carácter de proceso permanente al desarrollarse a través de toda la vida de los individuos y las colectividades por las vías formales, informales y no formales de la educación.

B) Pasa de educación encaminada a darle solución a los problemas del entorno inmediato, a educación encaminada a resolver los problemas del medio ambiente, manejándose el término de competencias asociado a la solución de esos problemas, no obstante, con un escaso tratamiento del abordaje didáctico y pedagógico de la formación de éstas.

La década de los ochenta coincide con el agravamiento y generalización de la crisis ambiental y con el correlativo incremento de la preocupación al respecto. Su carácter global se hace más evidente, y se enfatizan los aspectos relacionados con la desigualdad mundial y con los desequilibrios Norte-Sur. El Informe de Brundtland (1987) alerta sobre la necesidad imprescindible de vincular los problemas ambientales con la economía internacional y sobre todo con los modelos de desarrollo y aporta una concepción de desarrollo sostenible. 
A partir del reconocimiento de la educación ambiental como proceso encaminado a la formación de los individuos en materia ambiental, comienza a dársele tratamiento de dimensión de la educación, otorgándosele una especial importancia a su instrumentación por las vías formales de la educación, se reconoce además la necesidad de trabajar desde la perspectiva metodológica y didáctica su implementación.

En cuanto a la Educación Ambiental inicial de los maestros del nivel bàsico García Gómez R. en una aproximación a cómo debía ser la misma plantea que "Entendemos que si un profesor de enseñanza primaria debe integrar todas las diferentes disciplinas en torno al medio, su formación no puede ser sólo y claramente disciplinar y que sea él en el aula el que haga esfuerzos de integrar lo que ha recibido como disciplinar. Será preciso darle ya una formación integrada de las ciencias sociales y naturales en su dimensión medio ambiental". "Será necesario dotar al maestro de la formación suficiente para que sea capaz de seleccionar y secuenciar contenidos y elegir la metodología más apropiada a las características y procesos de aprendizajes de los alumnos. Este diseño curricular base para la enseñanza infantil y primaria representa un primer nivel de concreción y serán los centros y los maestros quienes desarrollen los siguientes niveles de concreción (1989: 473)

En ese orden, en el Ecuador el programa para la formación de docentes del nivel básico correspondiente a los años 1975 y 1983, contemplaba la didáctica de Ciencias Naturales dentro de su estructura curricular, donde se enfatiza privilegiar la naturaleza como recurso didáctico, y el desarrollo de una actitud científica sobre 
la base de los contenidos estrictamente de los elementos naturales. No se aborda la educación ambiental como objeto de estudio en la formación de profesores atendiendo a que al igual que en otros países del orbe y en especial de Latinoamérica, ésta era asumida por Organizaciones No Gubernamentales.

Tercera etapa: Redimensionamiento de la Educación Ambiental. (1992 hasta la actualidad).

A partir de la Conferencia Cumbre de la Tierra, celebrada en Río de Janeiro, Brasil (1992) se amplía la concepción de la educación ambiental, proyectándose como uno de los elementos esenciales para el logro del desarrollo sostenible.

Entendiéndose por desarrollo sostenible "la preservación del medio ambiente para las actuales y futuras generaciones". En ese orden Novo M. (2009) considera que "El desarrollo sostenible, es el proceso que, inspirado por un nuevo paradigma, nos orienta sobre los cambios que hemos de practicar en nuestros valores, formas de gestión, criterios económicos, ecológicos y sociales, para mitigar la situación de cambio global en que nos encontramos"... (2009: 199)

Así, en la Agenda 21, que es uno de los documentos principales de los aprobados en dicho foro, su capítulo 36 plantea que "Cuando hablamos de Educación Ambiental estamos haciendo referencia a un proceso sostenido donde todos y cada uno de los integrantes de la comunidad toman conciencia de su medio y adquieren conocimientos, valores y competencias que contribuyen a una mayor y mejor calidad de vida". 
Es indiscutible que la educación ambiental en esta etapa adquiere una nueva dimensión que influye en su tratamiento e instrumentación. Significa la preparación de los individuos y las comunidades no sólo para preservar el medio ambiente, sino hacerlo de manera armónica con el crecimiento económico, el mejoramiento social y la calidad de vida.

En esta etapa se le confiere especial atención en los foros y conferencias internacionales a los aspectos didácticos y metodológicos de la educación ambiental. Se insiste en la necesidad de la búsqueda de nuevos enfoques y métodos para su implementación; la integración de todas las disciplinas; el desarrollo de habilidades en la solución de problemas ambientales sobre la base de situaciones reales; la vinculación a la práctica, como vías para lograr mejores resultados en la adquisición y desarrollo en los sujetos en formación de conocimientos, habilidades y valores con respecto al medio ambiente.

Así, en la Conferencia Mundial sobre Educación para Todos, organizada por la UNESCO y el PNUMA, 1990, Thailandia. Se hace alusión a que debe comprenderse que: "Para iniciar la educación ambiental no es una cuestión sencilla de incluir una serie de ajustes a los programas tradicionales, sino más bien convocar a nuevos enfoques, métodos y contenidos". Revistiendo una vital importancia la solución de problemas relacionados con el medio ambiente.

La propia Agenda 21 refiere que "Para ser eficaz, la educación en materia del medio ambiente y desarrollo debe ocuparse de la dinámica del medio físico-biológico, del medio socioeconómico y el desarrollo humano e integrarse en todas las disciplinas". 
En el Congreso Mundial de Educación y Comunicación sobre Ambiente y Desarrollo (ECOED), Organizado por la UNESCO, la Cámara de Comercio Internacional (ICC), el PNUMA, la Organización de Educadores Ambientales de Estados Unidos (NAAEE) y Canadá (COEO), 1992,celebrado en Toronto, Canadá, se hace referencia a que " Hay que enfocar los problemas reales, identificar las soluciones prácticas, plantear preguntas éticas y utilizar las habilidades del pensamiento crítico, así como las habilidades intuitivas y de apreciación" . Esta educación debe considerar y desarrollar cualidades y valores éticos y morales de la personalidad.

Según Romañá, (1994) Citado por Novo M. (2009), “En cuanto a las vías que es preciso utilizar para promover la E.A., los medios y estrategias son múltiples y están desarrollados en numerosos textos y manuales, pero tal vez convenga recordar que «educación ambiental» equivale a «educación para la competencia ambiental» e incluye aspectos no sólo cognoscitivos, sino también afectivos, axiológicos y «estéticos» (1994: 14).

En cumplimiento del mandato realizado por la UNESCO en la Conferencia de Moscú (1987) sobre la necesidad de impulsar Estrategias Nacionales y Regionales de Educación Ambiental, los diferentes países y comunidades comenzaron a elaborar este tipo de documentos durante la década de los años noventa.( Novo M. :2009) Comienza así, con la reforma de los planes de estudios desarrollada en ese período la inclusión de la dimensión ambiental en las diferentes carreras universitarias, incluyendo aquellas que están encaminadas a la formación de los docentes. 
En ese orden, se aprecia que países como Cuba, México, Colombia, España, Brasil, Chile Argentina, entre otros, comienzan a introducir la Educación Ambiental en la malla curricular de la formación inicial de docentes.

En el caso del Ecuador, en el año 1994, el MEC, la CAAM, la UNESCO y Ecociencia elaboraron la agenda ecuatoriana de educación y comunicación ambiental para el desarrollo sustentable, en la que se establecen consensos, lineamientos y políticas de acción y como resultado de este proceso el Ministerio de Educación y Cultura, mediante acuerdo ministerial 2188 de del 25 de Abril de 1995, aprobó el "Reglamento de Políticas de educación, Capacitación y Comunicación Ambiental" que reconoce la importancia de incorporar la educación ambiental en la educación formal, en todos los niveles educativos, incluyéndola en los planes y programas de estudio sin requerir de una asignatura especial. Por lo tanto, la dimensión ambiental debe ser tratada como un eje transversal del currículo, con temas fundamentales que impregnan la actividad educativa y que acercan la escuela a la vida y favorecen el desarrollo integral de la persona.

Así, en la propuesta curricular aprobada en el año 2000, para la formación de los maestros del nivel básico, está en función del mejoramiento de la reforma curricular realizada en el año 1991 en muchos aspectos, estando dentro de ellos la formación de un perfil que incluye elementos del papel de este docente en el logro de la sustentabilidad y la protección al medio ambiente, perfeccionándose además los programas de las disciplinas de Sociología de la Educación y Didáctica de las Ciencias Naturales; no obstante, prevalece una visión eminentemente naturalista del medio ambiente, con elementos conservacionistas, y la formación está 
encaminada en lo esencial al desarrollo de proyectos comunitarios en función de la protección del medio, teniendo como sustento la Sociología de la Educación, cuestión que es loable, pero no suficiente para que una vez egresados de la carrera magisterial asuman la educación ambiental de sus alumnos en correspondencia con las exigencias internacionales y las del propio país.

Mediante la Declaración del Milenio de 2000, los 189 Estados Miembros de las Naciones Unidas se comprometieron a redoblar los esfuerzos para erradicar la pobreza en el mundo. Se establecieron así los Objetivos de Desarrollo del Milenio (ODM), cuyas metas deberán alcanzarse en 2015, tomando como año base 1990. La sostenibilidad ambiental se incluye en esos Objetivos debido a su importancia en la lucha contra la pobreza, la salud, la igualdad de género y los demás componentes del desarrollo.

Así, en diciembre del año 2002 se adoptó la decisión de declarar el decenio 20052014 como el Decenio de las Naciones Unidas de la Educación para el Desarrollo Sostenible mediante la Resolución 57/254 de la UNESCO, organización encargada de llevar a cabo la promoción de la Década, reconoce que no existe un modelo universal de educación para el desarrollo sostenible (EDS), por lo que hace un llamamiento generalizado a todos los movimientos educativos existentes (educación para la salud, para la interculturalidad, para el consumo responsable, para la paz...) a fin de que incorporen la dimensión de la sostenibilidad. (Novo M. Op.cit).

En ese orden, en el caso de la Cumbre sobre Desarrollo Sostenible celebrada del Río de Janeiro (Brasil) 20 a 22 de junio de 2012 Rio +20, en su Declaración Final 
en los apartado 230,233 y 235 se refuerza la necesidad de lograr la EDS en el nivel de Educación Superior, de la forma siguiente:

230. "Reconocemos que las generaciones más jóvenes son custodios del futuro, (...) Por tanto, decidimos mejorar la capacidad de nuestros sistemas educativos a fin de preparar a las personas para que puedan lograr el desarrollo sostenible, en particular mediante una mayor capacitación de los docentes, la elaboración de planes de estudio relativos a la sostenibilidad..."

233." Resolvemos promover la educación para el desarrollo sostenible e integrar el desarrollo sostenible de manera más activa en la educación más allá del Decenio de las Naciones Unidas de la Educación para el Desarrollo Sostenible".

235. "Subrayamos la importancia de prestar apoyo a las instituciones educativas, en particular las instituciones educativas superiores de los países en desarrollo, para que realicen investigaciones y logren innovaciones para el desarrollo sostenible, en particular en la esfera de la educación,... dirigidas a remediar las deficiencias en materia de aptitudes a fin de avanzar los objetivos nacionales de desarrollo sostenible".

Según el criterio de Novo M. (2009), la Educación para el Desarrollo Sostenible no se presenta como un programa nuevo, sino como una invitación a repensar las políticas educativas, los programas y las prácticas pedagógicas. Al pretender impregnarlo todo, no puede sino estimular a las distintas corrientes educativas para que introduzcan o refuercen los contenidos que hacen referencia a problemas de desarrollo y fomenten la deseable sostenibilidad en nuestras sociedades. 
En ese orden, para Novo "La condición omnicomprensiva de la EDS, y la forma en que abarca a campos tan diferentes como la igualdad de géneros, el medio ambiente, el sida, la enfermedad, la agricultura..., hacen de ella una propuesta generalizada de carácter transversal y no tanto un movimiento educativo propiamente dicho." (Op. Cit.: 201)

Así, se confirma en las propias palabras de UNESCO (2009) “El principal objetivo de la EDS es integrar los valores inherentes al desarrollo sostenible en todos los aspectos de la enseñanza (...) mediante todas las formas de educación...” (2009:1).

En el caso especifico de la Educación Ambiental, Novo destaca, que "desde sus inicios, la educación ambiental se constituyó como una genuina educación a favor de un nuevo modelo de desarrollo, y que esta cuestión ha estado siempre presente en su teoría y en sus prácticas. Un desarrollo que primero se llamó ecodesarrollo, después desarrollo endógeno y más tarde desarrollo sostenible, pero que los educadores ambientales fueron alumbrando y extendiendo siempre desde un empeño compartido: educar para el arte de vivir en armonía con la naturaleza y de distribuir de forma justa los recursos entre todos los seres humanos. Una visión que ha sido pionera entre los movimientos alternativos al modelo de crecimiento económico ilimitado y al mantenimiento de enormes brechas entre ricos y pobres a lo largo nuestra historia reciente. "(Op.cit.: 202) Posición está que se asume a los efectos de la presente investigación.

Con relación a la EA Leff (2005) plantea que “...es una educación para comprender la complejidad del mundo actual. No sólo es una educación ecológica, ni una visión holística de las interrelaciones entre objetos. La E A llama a relacionar los procesos 
naturales, físicos y biológicos y los procesos económicos, sociales y culturales como pilares del desarrollo sostenible; pero es más que eso: implica una educación sobre la complejidad del mundo generada por la intervención del conocimiento sobre el mundo y sobre la vida. La educación ambiental es la educación de una ética de la vida y de la sustentabilidad frente a la imposición de la racionalidad económica e instrumental de la modernidad" (Leff E.:17)

Por otra parte, comienzan en esta etapa a desarrollarse trabajos e investigaciones en diferentes contextos referidas a la Educación Ambiental de los docentes en formación, dentro de los que se destacan los realizados por Vega Marcote V. (2004), Moreno Latorre E. (2005), Manzanares Gavilán M. y Fuentes Martínez (2006), García Ruiz M. y Sánchez Hernández(2006), Quintero Díaz C (2007) Camarena Gómez B (2009), Aguilera González A. (2009), Calixto Flores R. (2010), Rodríguez Marín F. (2011), Rodríguez Prieto M. (2009) Bravo Chávez W. (2011), Sánchez Martínez A. (2012), entre otros.

De igual forma, aparecen trabajos referidos a la formación de competencias asociadas a la sostenibilidad en el nivel universitario, estando dentro de ellos los realizados por Vega Marcote y Álvarez Suárez (2005); Martínez Agut, Aznar Minguet, UII Solís, y Piñeiro (2007, 2010); Álvarez y Vega (2009); Aznar Minguet, UII Solís(2009); Bula Caraballo (2011), Espejel Rodríguez, Castillo Ramos y Martínez de la Fuente (2011), Amador Hidalgo y Arjona Fuentes (2012), los que abordan esencialmente la determinación de estas competencias y algunos aspectos desde la perspectiva didáctica y pedagógica. 
En cuanto a la formación de competencias relacionadas con la Educación Ambiental de los maestros en formación, en la inmersión bibliográfica realizada se aprecia un pobre tratamiento del tema, se destaca la investigación realizada por Mugarra MA, Novo M. y Bautista Cerro M.J (2008) donde se presenta una experiencia a ese fin, en la modalidad de formación a distancia.

Por su parte, en el contexto ecuatoriano, en el año 2006 se realiza una nueva reforma curricular en la carrera de maestros del nivel básico, la que está vigente en la actualidad, encaminada al perfeccionamiento del Plan de Estudios, donde se trabaja en el perfeccionamiento de la formación por competencias, y aparecen dentro del perfil del profesor de este nivel, las siguientes competencias asociadas al objeto de esta investigación:

- Incorporar los ejes transversales y de formación integral en la planificación curricular, dentro de los que se encuentra la Educación Ambiental.

- Desarrollar programas para la preservación, conservación de la biodiversidad.

En cuanto a la malla curricular aparecen las disciplinas Realidad Socioeconómica, Cultural y Ecológica del País, así como las Ciencias Naturales y su Didáctica, y Estudios Sociales y su Didáctica, ambas taller, las que abordan el medio ambiente y el desarrollo sustentable, se incorpora en la de Práctica Docente del IV - V y VI nivel (Año de servicio educativo rural obligatorio), no obstante, se observa ausencia de la metodología relacionada con la Educación Ambiental, unido a que aún 
cuando se declara como principio de la reforma la interdisciplinariedad, la concepción seguida es eminentemente disciplinar, a lo que se le suma la falta de preparación de los docentes para enfrentar dicha formación, manteniéndose como una educación encaminada a la preservación y conservación de la biodiversidad y no a la solución de los problemas ambientales existentes, lo que limita, según el criterio de la autora de esta investigación, la formación de las competencias antes declaradas.

En ese orden, en el Programa plurianual 2011-2013, del Ecuador, al analizar la situación de la EA como eje transversal se plantea "El órgano rector de la educación nacional, a través de la instancia responsable de la implementación de esta política, todavía no logra planear ni poner marcha estrategias efectivas de carácter nacional, para que se desarrolle sistemáticamente la educación ambiental en el aula. Todavía el eje transversal en el sistema educativo nacional no supera un accionar disperso, asociado a las efemérides ambientales. En cuanto a los proyectos ambientales de las escuelas, éstos se han restringido a la reforestación, manejo de desechos, reciclaje, producción de materiales y campañas de sensibilización. Todo ello no ha impactado en un aprendizaje que conduzca a la formación de ciudadanos y ciudadanas con conciencia ambiental y capacidad para liderar cambios en comportamiento de la comunidad educativa". (2010:37)

\section{Metodología}

Se trabajó con fuentes secundarias de información bibliográfica para relatar el recorrido histórico de la Educación Ambiental; para el análisis fáctico se recurrió a 
fuentes de información primaria que fueron los docentes en formación del cuarto nivel del Instituto Superior Pedagógico "23 de Octubre" de Montecristi, además los profesores que cumplen la función de orientadores de la práctica docente, la información fue recogida a través de una encuesta.

\section{Resultados y Discusión}

En una investigación realizada a los docentes en formación y profesores orientadores de práctica docente del nivel básico del Instituto Superior Pedagógico "23 de octubre" de Montecristi se evidenció los siguientes resultados:

- Los estudiantes muestran desconocimiento de qué es la Educación Ambiental y el Desarrollo Sostenible.

- Tienen una visión fragmentada del medio ambiente y su concepción es eminentemente naturalista, al circunscribir éste y sus problemas con elementos del medio natural.

- No logran articular el medio ambiente y la concepción de su desarrollo sostenible.

- La mayoría reconoce desconocer las características, los métodos y estrategias más adecuados para desarrollar la EA.

- Las actividades o tareas de educación ambiental que desarrollan durante la práctica docentes no tienen en cuenta las potencialidades de las asignaturas que imparten a ese fin, siendo fragmentadas y con un marcado carácter naturalista y conservacionista, ausentes de reflexión crítica sobre las causas generadoras de los problemas ambientales y sus posibles soluciones. 
- Ni en su formación, ni en la realización de tareas durante las prácticas docentes se logra la articulación de eje transversal de la Educación Ambiental para el desarrollo sostenible.

- La mayoría de los estudiantes reconocen que las competencias que han desarrollado durante la carrera no son suficientes para poder educar ambientalmente en la sostenibilidad a los alumnos del nivel básico.

Las opiniones vertidas por los investigados reflejan un pobre conocimiento respecto a la educación ambiental, a pesar de que se tiene la base legal, una constitución eminentemente ecologista y otros instrumentos legales, que conducen a los ciudadanos a crear una conciencia ecológica para el buen vivir; mas, en las instituciones educativas de formación docente no se lo está cumpliendo a satisfacción, lo que demanda interés y atención de la comunidad educativa de estos centros de estudios; en este sentido se comparte la opinión de Aznar Minguet (2005) que la educación para la sostenibilidad debería contemplar la formación de competencias básicas en tres ámbitos: el cognitivo: competencias cognitivas relacionadas con el saber y vinculadas con la comprensión crítica de la problemática medioambiental global y local; competencias metodológicas, relacionadas con el saber hacer, la adquisición de habilidades, estrategias, técnicas y procedimientos para la toma de decisiones y la realización de acciones relacionadas con el medio ambiente y el desarrollo sostenible; competencias actitudinales, relacionadas con el saber ser y valorar, donde el desarrollo de actitudes y valores de sostenibilidad resulta imprescindible.

\section{Conclusiones:}


- La educación ambiental en su concepción pasa de educación para resolver problemas del medio ambiente, a educación ambiental para la sustentabilidad.

- Se introduce paulatinamente la EA en la malla curricular de los maestros en formación y comienzan a desarrollarse investigaciones y trabajos relacionados con su implementación.

- Comienzan de manera incipiente a desarrollarse trabajos en distintos países referidos a la formación de competencias asociadas a la sostenibilidad en el nivel universitario y de los maestros en formación; no obstante, no está suficientemente abordado desde la perspectiva pedagógica la formación de competencias de Educación Ambiental para la sustentabilidad de los maestros del nivel básico en formación.

- En el contexto ecuatoriano la educación ambiental y la formación de competencias de educación ambiental para la sustentabilidad en los maestros del nivel básico en formación es aún un problema no resuelto.

- Los estudiantes de docencia muestran desconocimiento de qué es la Educación Ambiental y el Desarrollo Sostenible, tienen una visión fragmentada del medio ambiente y su concepción es eminentemente naturalista, desconocen las características, los métodos y estrategias más adecuados para desarrollar la EA.

\section{BIBLIOGRAFÍA}

AGUILERA GONZÁLEZ, Antonio. (2009). La Educación Ambiental de los profesionales en formación de la carrera Licenciatura en Educación especialidad Mecánica. Tesis presentada en opción al grado científico de Doctor en Ciencias Pedagógicas. Instituto Superior Pedagógico "José de la Luz y Caballero". Cuba. 
ÁLVAREZ PEDRO Y VEGA PEDRO (2009) Actitudes Ambientales y conductas sostenibles. Implicaciones para la educación ambiental. Revista Psicodidáctica, Vol. 14, Núm. 2. Pp245-260 ISSN (Versión impresa): 1136-1034 pebpanas@vc.ehu.es Universidad del País Vasco/Euskal Herriko Unibertsitatea España. Disponible en: http://redalyc.uaemex.mx/src/inicio/ArtPdfRed.jsp?iCve=17512724006.

AZNAR, Pilar y ULL, Mํㅡㄹ de los Ángeles. (2009). La formación de competencias básicas para el desarrollo sostenible: el papel de la Universidad. Revista de Educación, número extraordinario 2009, pp. 219-237. España. http://dialnet.unirioja.es/servlet/articulo?codigo=3019436

AZNAR MINGUET, Pilar. (2005). La escuela y el desarrollo humano sostenible: retos educativos a nivel local. Ediciones Universidad de Salamanca Teor. educ. 14, pp. $1 S 1$-1 S. España. ISSN: 1130-3743.

http://gredos.usal.es/jspui/handle/10366/71956

BRAVO CHÁVEZ, William. (2011). Estudio de los conocimientos de los profesores sobre educación ambiental. En: Revista de Didáctica Ambiental № 9. Páginas 5070. Chile.

BULA, Germán. (2011). Educación ambiental: del enfoque por competencias al enfoque por habilidades. ISSN: 2011-8643 | Vol. 5 | No. 10 | 2011 | pp. 33-42.

http://revistas.usta.edu.co/index.php/magistro/article/view/42

CAMARENA, Beatriz. (2009). Educación Ambiental y Formación de Profesorado en México: Estudio de Perfiles Ambientales en las Escuelas Normales de Sonora. Tesis Doctoral. Salamanca - España.

http://gredos.usal.es/jspui/handle/10366/76396

ESPEJEL, Adeline, et al. (2011). Modelo de educación ambiental para el nivel medio superior, en la región Puebla-Tlaxcala, México: un enfoque por competencia. Revista ibero-americana de educação ISSN: 1681-5653 № 55/4 - México. http://www.rieoei.org/expe/3705Espejel.pdf

GARCÍA RUIZ, Mayra. SANCHEZ HERNÁNDEZ, Beatriz. (2006). Las actitudes relacionadas con las ciencias naturales y sus repercusiones en la práctica docente de profesores de primaria. Vol. XXVIII, num. 114, pp.61-89. México. http://scielo.unam.mx/scielo.php?pid=s0185-26982006000400004\&script=sci arttext

FLORES, R. C. (2010). Medio ambiente y educación ambiental: representaciones sociales de los profesores en formación. magis, Revista Internacional de Investigación en Educación, 2 (4), 401-414.

dialnet.unirioja.es/descarga/articulo/3237836.pdf 
GONZALEZ MUÑOZ, María del Carmen. (1998). La educación ambiental y formación del profesorado. Revista Iberoamericana de Educación № 16, págs. 1322. España. http://dialnet.unirioja.es/servlet/articulo?codigo=1020277

HIDALGO, Luis y ARJONA, Juan (2012). La sostenibilidad curricular en el ámbito universitario y la adquisición de competencias básicas: capacitar para construir un futuro sostenible. Octavo Congreso Internacional sobre Educación, Cultura y Desarrollo. España. http:/www.eumed.net/eve/8ecd-cerradp/pon/ahaf.doc

MINISTERIO DE EDUCACIÓN Y CULTURA, CONSEJO NACIONAL DE EDUCACIÓN SUPERIOR. (2006). Formación Docente Inicial de Educación Básica en los Institutos Superiores Pedagógicos. Quito.

NOVO, María. (2009). La educación ambiental, una genuina educación para el desarrollo sostenible. Revista de Educación, número extraordinario, pp. 195-217. España. http://dialnet.unirioja.es/servlet/articulo?codigo=3019430

NUÑEZ MORALES, Marylú. (2003). Modelo pedagógico para educar en el valor responsabilidad ambiental a los estudiantes de la carrera de derecho. Tesis presentada en opción al grado científico de Doctor en Ciencias Pedagógicas. Universidad de Camagüey Centro de Estudios de Ciencias de la Educación "Enrique José Varona". Cuba.

VALDÉS, ORESTES. (2002) La evaluación de la educación ambiental desde una concepción teórico-práctica interdisciplinaria e integradora en los programas y proyectos educativos institucionales en Cuba.

www.unesco.org/.../

VEGA MARCOTE, Pedro. (2004). La educación ambiental en la formación inicial del profesorado. Análisis de un modelo didáctico para el desarrollo de la competencia para la acción a favor del medio. Universidad de A Coruña. España. http://ruc.udc.es/dspace/handle/2183/1113

MARTÍNEZ AGUT, et al. (2010) Análisis de la introducción de la sostenibilidad en la enseñanza superior en Europa: compromisos institucionales y propuestas curriculares Revista Eureka sobre Enseñanza y Divulgación de las Ciencias 2010, 7, № Extraordinario, pp. 413-432. Asociación de Profesores Amigos de la CienciaEureka. ISSN: 1697-011X. DL: CA-757/2003 http://www.apac-eureka.org/revista.

MANZANARES, Mercedes. FUENTES, Andrés. (2006). Percepciones sobre desarrollo sostenible de los maestros en formación inicial. I Congreso Iberoamericano de Ciencia, Tecnología, Sociedad e Innovación CTS+I. Argentina. http://www.oei.es/memoriasctsi/mesa16/m16p10.pdf 
MORENO LATORRE, Esther. (2005). La formación inicial en educación ambiental de los profesores de secundaria en período formativo. Tesis Doctoral. Universidad de Valencia. España. http://roderic.uv.es/handle/10550/15334

RODRÍGUEZ MARÍN, Fátima. (2011). Educación ambiental para la acción ciudadana: concepciones del profesorado en formación sobre la problemática de la energía. Tesis para optar por el grado de Doctora. Universidad de Sevilla. España. http://dialnet.unirioja.es/servlet/tesis?codigo=23901

SÁNCHEZ MARTINEZ, Armando. (2012). Retos y perspectivas de la educación ambiental en la educación básica. III Foro Nacional de Educación Ambiental ciudad de Veracruz. México.

http://anez.org.mx/docs/Retosyperspectivas\%20EAEBASM.pdf

VEGA, PEDRO Y ÁLVAREZ PEDRO (2005) Planteamiento de un marco teórico de la Educación Ambiental para un desarrollo sostenible. Revista Electrónica de Enseñanza de las Ciencias Vol. 4 № 1. www.saum.uvigo.es/reec/.../ART4_Vol4_N1.pdf 